Rivett, and Prof. O. U. Vonwiller to advise it as to (1) the locations of the two to be retained; (2) the disposal of existing equipment; and (3) the probable initial cost, if any, of supplementing existing equipment at the two chosen observatories, the estimated cost of maintenance of these two, and the best means of disposing of outstanding work in connexion with the Astrographic Catalogue, to which work several of the States were committed many years ago. Maybe two institutions, if thoroughly well supported, will be more effective than five inadequately maintained; but many will view with misgiving a decision of this kind based mainly on a desire to save money.

\section{Early Man in East Africa}

The conclusions at which Dr. Leakey, Dr. H. Reck, and Mr. A. T. Hopwood arrived in their recent investigation of the Oldoway Beds in relation to the antiquity of the human skeleton found in these deposits would appear to have received confirmation by a find at Kanam, a small native village on Lake Victoria. Here Dr. Leakey, it is reported in a dispatch from Nairobi which appeared in the Times of April 19, has discovered a lower jaw of Homo sapiens in deposits containing pre-Chellean implements and teeth of the same species of Deinotherium as was discovered at Oldoway. At Kanam, however, the beds in which the find was made correspond with the oldest of the Oldoway beds, whereas Oldoway man was found in Bed No. 2. Other finds reported from early Pleistocene deposits at Kanam are fragments of three human skulls, apparently washed out from deposits containing Chellean tools and remains of Elephas antiquus and Hipparion. At first sight, this find on Lake Victoria carries Homo sapiens back a stage further than the Oldoway discovery; but a more detailed account of the deposits and the conditions of the find must be awaited before a certain conclusion is possible. The difficulty of accepting a skeleton found entire, as was Oldoway man, as contemporary with the alluvial deposits in which it occurs - a difficulty already pointed out (NATURE, Feb. 27, p. 312)-is stressed in the current number of L'Anthropologie (t. 42, p. 214), where it is also suggested that the general conformation of the Oldoway skull points to an affinity with the modern Masai.

\section{Liquid Carbon Dioxide in Ocean Water}

IN an interesting article (C.R. Acad. Sci. Leningrad, 1931), the eminent Russian geochemist, V. Vernadsky, directs attention to the fact that the conditions of temperature and pressure in the depths of oceans should necessitate the existence there of carbon dioxide in a stable liquid state. The chemical inter. relations of water and liquid carbon dioxide are as yet unknown, but they must play an important part in the life of organisms at great depths. Their gaseous metabolism should differ greatly from that of organisms inhabiting the surface layers of water, where the expired carbon dioxide can exist as a gas. It is known that the lower limit of plankton is about 200 metres from the surface, while sunrays penetrate very much deeper, and photo-synthesis is theoretically possible for red algæ down to 400-500 metres. They do not, however, occur below 200 metres, which means that the limit of their distribution is not governed by the lack of active light rays, but probably coincides with the zone where the gaseous state of carbon dioxide becomes unstable and the liquid state appears. Again, the conditions of gaseous metabolism of numerous organisms in the mud at the bottom of the oceans should be highly peculiar, the gases present there constituting an atmosphere very different from that in the mud of shallow waters. The interesting fact discovered more than a hundred years ago by Biot that the bladder of deep-sea fishes contains pure oxygen, which, according to later discoveries, is produced by special glands, has always remained a physiological puzzle, but it is possible that this is also connected with their life in the zone where only liquid carbon dioxide exists. It is legitimate to ask, for example, whether the oxygen in the bladder is not the result of decomposition of liquid carbon dioxide. All these considerations and examples serve to stress the necessity of organising systematic investigations on the distribution of gaseous and liquid carbon dioxide in different zones of oceans.

\section{The Willis Navigating Machine}

Aт a recent meeting of the Royal Astronomical Society, the capabilities of this ingenious instrument (aviation type), designed by Mr. E. Willis for the solution of spherical triangles, were demonstrated by Dr. L. J. Comrie, Superintendent, H.M. Nautical Almanac Office. Mechanically, the instrument is a combination of an alt-azimuth and equatorial with five graduated circles corresponding to altitude, azimuth, latitude, declination, and hour angle. The principal problem in nautical astronomy is the calculation of the altitude and azimuth of a heavenly body from given values of the latitude, declination, and hour angle. These quantities are set on the corresponding circles and the machine at once gives the appropriate altitude and azimuth, which can then be used, in conjunction with the altitude from a sextant observation, to give the position line on the chart. This is only one of the many problems that the instrument can solve. In the type of instrument designed for aero. nautical navigation, the circles read to five minutes of arc, which is sufficiently accurate in practice, being of the same order of accuracy as readings made with a bubble-sextant. The instrument designed for marine purposes is larger and more accurate; in this type the graduated circles read to one minute of arc. In æronautical navigation, the quick reduction of observations is an essential desideratum, and the Willis machine gives complete satisfaction in this respect. The instruments are manufactured by Messrs. Heath and Co., New Eltham, London, S.E.9.

\section{Insulators for Switchgear and Busbars}

AT present there is a great demand for insulators for high voltage outdoor isolating switchgear. With a pressure of 132,000 volts and a high mechanical stress, the greatest care has to be used in their design, as any breakdown in the continuity of supply of a

No. 3260 , VoL. 129] 